\title{
Antitumor DNA vaccination against the Sox2 transcription factor
}

\author{
INGRID POLAKOVA, MARTINA DUSKOVA and MICHAL SMAHEL
}

\author{
Department of Experimental Virology, Institute of Hematology and Blood Transfusion, 12820 Prague 2, Czech Republic
}

Received January 22, 2014; Accepted March 17, 2014

DOI: 10.3892/ijo.2014.2402

\begin{abstract}
As cancer stem cells (CSCs) are resistant to chemotherapy, radiotherapy and targeted molecular therapy, immunotherapy of tumors could be aimed at their elimination. Markers specific for CSCs have not been identified to date, but microarray analyses have shown that CSCs and embryonic stem cells use similar transcriptional programs, thus suggesting the production of shared transcription factors. In this study, we developed an experimental DNA vaccine against the transcription factor Sox 2 that is important for self-renewal of stem cells and is overexpressed in numerous human cancers. The Sox 2 gene was codon optimized for the expression in human cells, its sequences encoding two nuclear localization signals (NLSs) were mutagenized, and the sequence coding for the PADRE helper epitope was fused with its 5 ' terminus. While codon optimization did not increase Sox 2 production and mutagenesis in NLSs only partially reduced nuclear localization of Sox 2 , the addition of the PADRE epitope was crucial for the enhancement of Sox 2 immunogenicity. The antitumor effect was shown after immunization against mouse oncogenic TC-1/B7 cells derived from the lung cancer cell line TC-1 and characterized by high Sox 2 production. Sox 2 -specific reactivity in an ELISPOT assay was further augmented by the depletion of regulatory $\mathrm{T}$ (Treg) cells, but this depletion did not enhance the antitumor effect. These data demonstrated the induction of immune responses against the Sox 2 self-antigen, but did not confirm the usefulness of Treg depletion when combined with antitumor vaccination.
\end{abstract}

\section{Introduction}

Cancer stem cells (CSCs) have been identified in numerous human cancers including both hematological malignancies and solid tumors. These pluripotent cells with unlimited proliferative potential, which are characterized by the ability to self-renew and differentiate, constitute a subset of cells within

Correspondence to: Dr Michal Smahel, Department of Experimental Virology, Institute of Hematology and Blood Transfusion, U Nemocnice 1, 12820 Prague 2, Czech Republic E-mail: smahel@uhkt.cz

Key words: Sox2, DNA vaccine, helper epitope, Treg depletion a tumor capable to initiate and propagate tumor growth (1). Therefore, to cure patients with malignant diseases, CSCs need to be eradicated. Unfortunately, CSCs are resistant to chemotherapy, radiotherapy, and targeted molecular therapy due to slow proliferation and production of membrane transporter proteins and detoxifying enzymes. As CSCs can express high level of MHC class I molecules and tumor antigens (2), immunization against CSC markers might be an important method of CSC elimination. To identify CSCs, cell surface markers are usually utilized, but unique CSC-specific markers are still missing.

Gene expression profiling of human stem cells has identified different expression programs for adult and embryonic stem cells (ESC) $(3,4)$. It has also shown a shared transcriptional program of ESCs and CSCs (3). Moreover, ESC-like expression has been found in aggressive human tumors (5). These observations suggest that the regulatory network controlling the function of ESCs may also be active in CSCs. The key regulators of ESC identity including self-renewal are the transcription factors OCT4, SOX2, and NANOG that co-operate in the regulation of transcription of large sets of genes (6). Production of these transcription factors has also been shown in various human tumors. For instance, SOX2 overexpression was proved in small cell lung cancer (7), monoclonal gammopathy, multiple myeloma (8), glioblastoma $(2,9)$, Merkel cell carcinoma, melanoma (10), gastric carcinoma (11), and breast carcinoma (12). Amplification of the SOX2 gene was found in a proportion of squamous cell carcinomas (SCC) of various organ locations including the esophagus, lung (13-15), oral cavity (16), uterine cervix, skin and penis (17). In SCC of the lung, SOX2 was identified as a lineage survival oncogene $(13,15)$ and its overexpression was associated with favorable prognosis (18) while in most other cancers, high SOX2 expression was predictive of poor prognosis.

SOX2 is a high mobility group (HMG) domain protein that contains two nuclear localization signals (NLS), a bipartite NLS motif and a basic cluster NLS motif, that are conserved in the HMG domain of transcription factors (19). This protein is highly immunogenic, both humoral and cell-mediated immune reactions were demonstrated in patients with premalignant and malignant diseases $(7,8)$. Moreover, the activation of SOX2-specific $\mathrm{CD}^{+} \mathrm{T}$ lymphocytes was shown after immunization with dendritic cells primed with apoptotic CSCs (2). SOX2 plays a pivotal role in the maintenance of stemness and tumorigenicity of CSCs and is also involved in cell proliferation, growth, migration, and chemoresistance 
(reviewed in ref. 20). Consequently, the SOX2 protein may be an appropriate target for tumor therapy $(9,21)$.

In this study, we modified the Sox 2 gene and constructed DNA vaccines against the Sox 2 protein, determined the immunogenicity of these vaccines, and utilized the depletion of regulatory $\mathrm{T}$ lymphocytes (Treg) for the enhancement of vaccine efficiency. We also characterized Sox 2 expression in the mouse oncogenic TC-1 cell line and its clones and demonstrated the antitumor effect against TC-1/B7 cells that was not augmented by Treg depletion.

\section{Materials and methods}

Plasmids. The wild-type (wt) Sox 2 gene was isolated from the F9 mouse testicular teratoma cell line (obtained from CLC, Eppelheim, Germany). Total RNA was extracted from these cells by the RNeasy kit (Qiagen, Hilden, Germany) and after the treatment with DNase (Promega, Madison, WI, USA), a reverse transcription was performed with the M-MLV reverse transcriptase (Promega). The Sox 2 cDNA was amplified by PCR using the Phusion High-Fidelity DNA Polymerase (Finnzymes, Espoo, Finland) and the Sox2-derived primers 5'-GTCTCGA GCCGCCATGTATAACATGATGGAGA-3' (forward) and 5'-GTCTCGAGTCACATGTGCGACAGGGGCA-3' (reverse) with the Kozak sequence and XhoI restriction sites added. Subsequently, the PCR product was cloned into the XhoI site of the pBSC plasmid (22). The Sox2opt gene with codons optimized for expression in human cells and the Kozak sequence added was designed and synthesized by GeneArt (Regensburg, Germany) and cloned into the EcoRI site of the pBSC plasmid. Then, the Sox2opt gene was mutated with the GeneEditor in vitro Site-Directed Mutagenesis system (Promega). To abolish the two NLSs present in Sox2 (19), altogether six nucleotides were changed in one reaction using three mutagenic oligonucleotides (Fig. 1). This mutagenesis resulted in six substitutions in the amino acid sequence. The substitutions K43T together with R44G and R57L together with R58G in the first NLS (NLS1) were created using the oligonucleotides 5'-ATCGGGTGAㅡAGGGCCTATG-3' and 5'-AGGCCAGCTAGGAAAAATGG-3' (the mutated nucleotides are underlined), respectively, and the substitutions R114L and R115G in the second NLS (NLS2) were introduced using the 5'-ACCGACCTCTAGGGAAAACC-3' mutagenic oligonucleotide. The resultant gene Sox2opt-cyt was amplified with primers 5'-TCGTCTCGAGTACAATATGATGGAAACCG-3' (forward) and 5'-TCGTCTCGAGTTAGCTAGCCATGTGAG ACAGAGGCAG-3' (reverse) and cloned into the XhoI site of the pBSC/Kozak+ATG plasmid that contains the Kozak sequence, NsiI site, XhoI site, and STOP codon inserted in the EcoRI site of pBSC. Into the NsiI site, the Pan DR epitope (PADRE) (23) was cloned using annealed oligonucleotides 5'-TGCCAAGTTCGTGGCTGCCTGGACCCTGAAGGCTG CCGCTATGCA-3' and 5'-TAGCGGCAGCCTTCAGGGTCC AGGCAGCCACGAACTTGGCATGCA-3', thus generating the PADRE.Sox2opt-cyt gene.

The sequence of all constructed genes was verified by DNA sequencing. Plasmids were propagated in E. coli XL1-blue strain cultured in Luria Broth medium with $100 \mu \mathrm{g} / \mathrm{ml}$ of ampicillin added and purified with the Qiagen Plasmid Maxi kit (Qiagen).
Peptides. The PADRE peptide (AKFVAAWTLKAAA; $>90 \%$ pure) was custom synthesized by GenScript (Piscataway, NJ, USA). The PepMix Human SOX2 (JPT Peptide Technologies, Berlin, Germany) is a mixture of 77 peptides covering the SOX2 protein ( $>70 \%$ pure; 15 -mers with an overlap of 11 amino acids).

Cell lines. NIH/3T3 fibroblasts established from mouse embryo culture (24) were obtained from the German National Resource Centre for Biological Material. TC-1 cells (provided by T.-C. Wu, Johns Hopkins University, Baltimore, MD, USA) were prepared by transformation of primary C57BL/6 mouse lung cells with the activated $\mathrm{H}$-ras and human papillomavirus type 16 (HPV16) E6/E7 oncogenes (25). TC-1/B7 cell line was derived from a TC-1-induced tumor formed in a mouse immunized against the E7 oncoprotein (26). All cells were grown in Dulbecco's modified Eagle's medium (DMEM; PAA Laboratories, Linz, Austria) supplemented with $10 \%$ fetal calf serum, $2 \mathrm{mM}$ L-glutamine, $100 \mathrm{U} / \mathrm{ml}$ penicillin, and $100 \mu \mathrm{g} / \mathrm{ml}$ streptomycin (PAA Laboratories).

Mice. Seven- to eight-week-old female C57BL/6 mice (Charles River, Sulzfeld, Germany) were used in immunization experiments. Animals were maintained under standard conditions and in accordance with the guidelines for the proper treatment of laboratory animals at the Center for Experimental Biomodels, Charles University, Prague. Animal protocols were approved by the First Faculty of Medicine Animal Use Committee.

Immunoblot staining. To verify the expression of the prepared genes, NIH/3T3 cells seeded in 4-cm dishes were transfected with $2.5 \mu \mathrm{g}$ of plasmid DNA using the Lipofectamine 2000 Transfection Reagent (Invitrogen, Carlsbad, CA, USA). Cotransfection with $0.5 \mu \mathrm{g}$ of the $\beta$-galactosidase encoding plasmid that served as an internal control of the transfection efficacy was conducted. After two days, the cells were collected and lysed in a modified Laemmli lysis buffer (4\% sodium dodecyl sulfate, $20 \%$ glycerol, $10 \%$ mercaptoethanol, $2 \mathrm{mM}$ EDTA, 100 mM Tris, pH 8.0) (27). Proteins from cell lysates were separated by $10 \%$ sodium dodecyl sulfate/polyacrylamide gel electrophoresis (SDS-PAGE) and electroblotted onto a polyvinylidene difluoride (PVDF) membrane (GE Healthcare, Little Chalfont, UK). The membrane was blocked with $10 \%$ non-fat milk in PBS and incubated with Sox2 (clone L1D6A2; Cell Signaling Technology, Danvers, MA, USA) and $\beta$-galactosidase (Promega) mouse monoclonal antibodies. Subsequently, the membrane was stained with horseradishperoxidase-conjugated anti-mouse IgG secondary antibody (GE Healthcare). The blots were stained using the ECL Prime Western Blotting Detection reagent (GE Healthcare) and scanned by the UVP EC3 Imaging System (UVP, Upland, CA, USA).

For the determination of the Sox 2 production in cell lines, proteins corresponding to $2 \times 10^{5}$ cells were analyzed by SDS-PAGE and stained as described above. $\beta$-tubulin was also stained with rabbit antibodies (Sigma-Aldrich, St. Louis, MO, USA) for comparison of protein load.

Immunofluorescence staining. The NIH/3T3 cells grown on 8-well Lab-Tek II Chamber Slides (Thermo Fisher Scientific, 


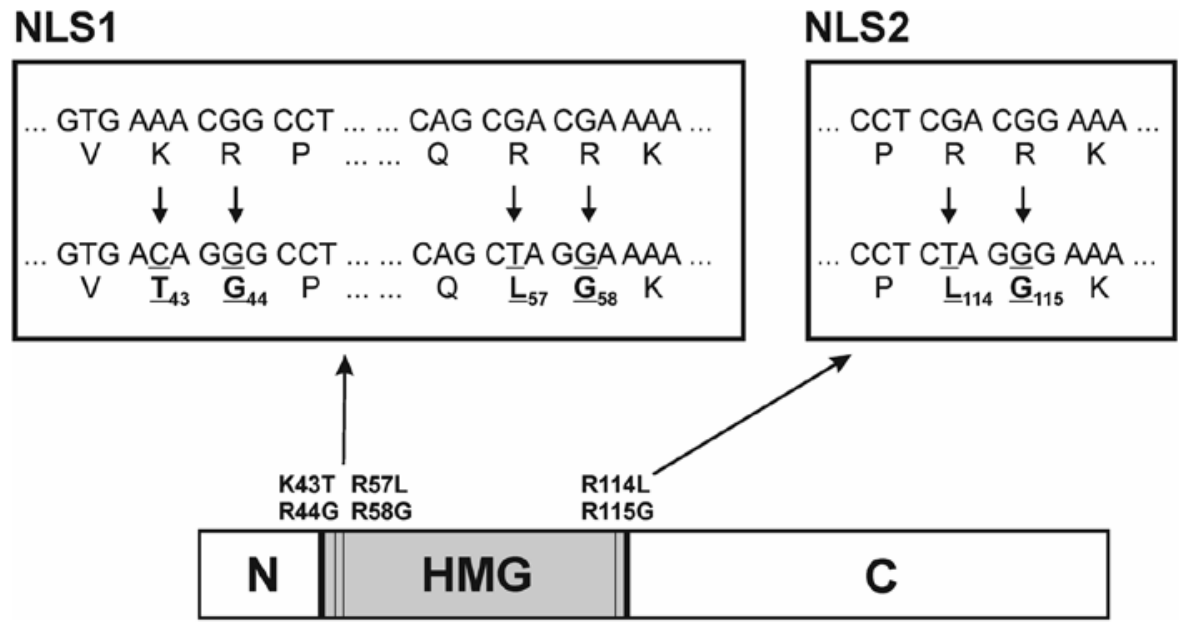

Figure 1. Mutagenesis in the NLSs of the Sox2opt gene. The modified positions are underlined. N, N-terminal domain, C, C-terminal domain.

Waltham, MA, USA) were transfected with $0.4 \mu \mathrm{g}$ of plasmids using Lipofectamine 2000 (Invitrogen). Two days after transfection, cells were fixed and permeabilized with methanol at $-20^{\circ} \mathrm{C}$ for $10 \mathrm{~min}$. The Sox 2 protein was stained with mouse monoclonal antibody (clone L1D6A2) followed by Alexa Fluor 488-conjugated goat anti-mouse IgG antibodies (Invitrogen). The nuclei of the cells were labeled with DAPI. The slides were examined by an Eclipse E600 microscope (Nikon, Tokyo, Japan).

Preparation of cartridges for the gene gun. Plasmid DNA was coated onto $1-\mu \mathrm{m}$ gold particles (Bio-Rad, Hercules, CA, USA) by the procedure recommended by the manufacturer. Each cartridge contained $1 \mu \mathrm{g}$ DNA coated onto $0.5 \mathrm{mg}$ of gold particles.

Immunization experiments. Mice were immunized with three $2-\mu \mathrm{g}$ doses of plasmid DNA at 1-week intervals by the gene gun (Bio-Rad). Vaccines were delivered into the shaven skin of the abdomen at a discharge pressure of 400 psi. One week after the last vaccination, pools of mononuclear cells were isolated from splenocytes (3 mice per group) using Ficoll-Paque (GE Healthcare) and analyzed by an ELISPOT assay or mice were s.c. inoculated with $3 \times 10^{4} \mathrm{TC}-1 / \mathrm{B} 7$ cells into the back under anesthesia with intraperitoneal (i.p.) etomidate $(0.5 \mathrm{mg} /$ mouse; Janssen Pharmaceutica, Beerse, Belgium). The tumor growth in the challenged animals was monitored twice a week and the tumor size was calculated from three perpendicular measurements using the formula $(\pi / 6)(\mathrm{a} \times \mathrm{b} \times \mathrm{c})$. In some experiments, $200 \mu \mathrm{g}$ of anti-CD25 antibody (clone PC61; Bio X Cell, West Lebanon, NH, USA) was i.p. inoculated four days before the first immunization.

ELISPOT assay. For detection of IFN- $\gamma$, multiScreen 96-well filtration plates with a PVDF membrane (Millipore, Billerica, MA, USA) were coated with $10 \mu \mathrm{g} / \mathrm{ml}$ of rat anti-mouse IFN- $\gamma$ antibody (BD Biosciences Pharmingen, San Diego, CA, USA) in $50 \mu \mathrm{l}$ of PBS and incubated overnight at $4^{\circ} \mathrm{C}$. Mononuclear cells resuspended in serum-free CTL-Test medium (Cellular Technology Ltd., Shaker Heights, OH, USA) were added to the plate $\left(8 \times 10^{5} /\right.$ well $)$ and incubated at $37^{\circ} \mathrm{C}$ in $5 \% \mathrm{CO}_{2}$ for $20 \mathrm{~h}$ either with or without $1 \mu \mathrm{g} / \mathrm{ml}$ of SOX2-derived pool of peptides (PepMix Human SOX2) or $1 \mu \mathrm{g} / \mathrm{ml}$ of the PADRE peptide. The cells were removed by three washes with PBS and three washes with PBS- $0.05 \%$ Tween-20. Then, $4 \mu \mathrm{g} / \mathrm{ml}$ of biotinylated rat anti-mouse IFN- $\gamma$ antibody (BD Biosciences Pharmingen) in $50 \mu \mathrm{l}$ PBS were added per well and cultured at $4{ }^{\circ} \mathrm{C}$ overnight. The wells were washed four times with PBS- $0.05 \%$ Tween-20 and incubated for $30 \mathrm{~min}$ with $50 \mu \mathrm{l}$ of 1:100 dilution of streptavidin-horseradish peroxidase (BD Biosciences Pharmingen) in PBS at room temperature. After washing three times with PBS-0.05\% Tween-20, followed by three washing steps with PBS alone, the spots were developed using the AEC Substrate Set (BD Biosciences Pharmingen). The induced spots were counted by a CTL-ImmunoSpot S5 UV Analyzer (Cellular Technology Ltd.).

Statistical analysis. The specific activation of mononuclear cells in ELISPOT was determined by Student's t-test. Tumor growth after DNA immunization was evaluated by the two-way analysis of variance and Bonferroni post-tests. A difference between groups was considered significant if $\mathrm{P}<0.05$. Calculations were performed using the Prism software, version 5.02 (Graph-Pad Software, San Diego, CA, USA).

\section{Results}

Construction of DNA vaccines. To increase potentially the production of the Sox 2 protein, we ordered a codon-optimized Sox 2 gene (Sox2opt) from the GeneArt Co. and cloned it into the pBSC plasmid. The expression of the optimized gene was evaluated by immunoblotting after transfection of NIH/3T3 cells. The steady-state level of the Sox 2 protein from the codon-optimized gene was comparable with that of the wt Sox 2 gene (Fig. 2).

As another step in the modification of the Sox 2 gene, we attempted to prevent the transcriptional activity of the Sox 2 protein by the abolition of its two NLSs. This alteration should increase the safety of a DNA vaccine against Sox2. In previous studies, only mutational inactivation of both NLS motifs in 


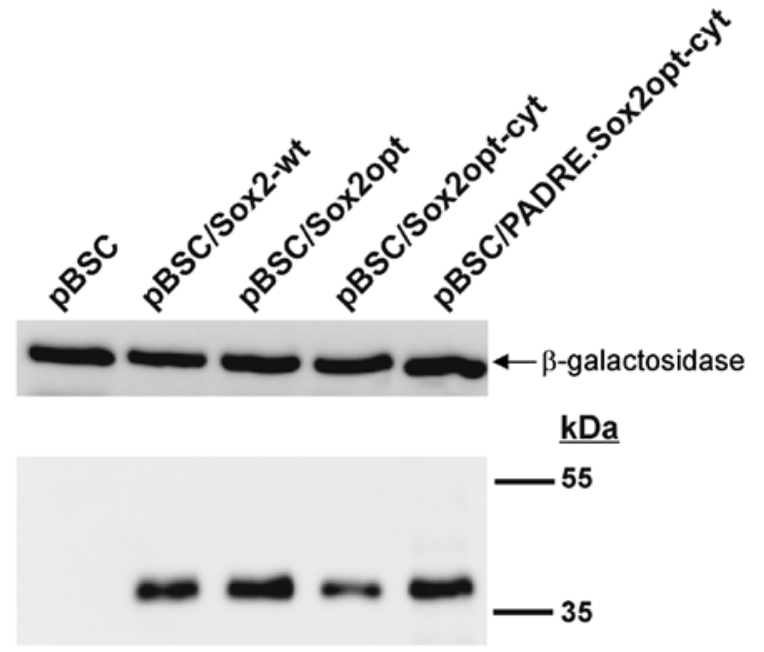

Figure 2. Production of the Sox 2 proteins. Two days after co-transfection of NIH/3T3 cells, Sox 2 production was analyzed by immunoblotting. To monitor transfection efficiency, $\beta$-galactosidase was also stained.

the HMG domain led to complete cytoplasmic localization of the SRY and SOX9 proteins (28) and analogous mutations in the Sox 2 transcription factor substantially reduced its nuclear localization (19). The site-directed mutagenesis of the Sox2opt gene substituting six nucleotides in the sequences corresponding to the two NLSs of the Sox 2 protein resulted in the Sox2opt-cyt gene (Fig. 1).

The expression of the Sox2opt-cyt gene was verified after transfection of NIH/3T3 cells by immunoblot (Fig. 2) and immunofluorescence analyses (Fig. 3). Immunofluorescence staining of transfected cells showed that the mutations in the NLS motifs reduced the ability of the Sox 2 protein to translocate to the nucleus, but besides localization in the cytoplasm, the Sox 2 protein was still present in the nuclei of most cells.
Finally, to support the immunogenicity of the DNA vaccine by the activation of $\mathrm{T}$ helper $(\mathrm{Th})$ cells, we fused the sequence encoding the strong PADRE helper epitope with the 5 ' terminus of the Sox2opt-cyt gene. The production (Fig. 2) of the fusion protein was comparable with that of the parental Sox2opt-cyt protein. The addition of PADRE did not change the mobility of the protein in SDS-PAGE, but the induction of the PADRE-specific response (see below) proves that the fusion protein was really synthesized. We also observed this phenomenon after the fusion of PADRE with the modified E7 oncoprotein (E7GGG). After immunofluorescent staining of the transfected cells, we found nuclei with highly variable intensity of labeling. The cytoplasm of the positive cells was uniformly stained (Fig. 3).

The PADRE epitope enhanced the immunogenicity of the DNA vaccine. The immunogenicity of the modified Sox 2 genes was examined after gene gun immunization of C57BL/6 mice with plasmid DNA. The IFN- $\gamma$-producing cells were detected by an ELISPOT assay after incubation with a pool of SOX2 peptides (PepMix SOX2). While no response was found after vaccination with the Sox2opt-cyt gene, the PADRE.Sox2opt-cyt gene induced a weak Sox 2-specific immunity (Fig. 4A). The high response shown after the stimulation with the PADRE peptide (Fig. 4B) suggests that activation of Th cells enhanced immunity against Sox 2 .

Depletion of Treg cells enhanced the efficacy of DNA vaccination. To further augment the weak Sox2-specific immunity, we depleted Treg lymphocytes with anti-CD25 antibody delivered four days before the first immunization. The stimulation of mononuclear cells with the PepMix SOX2 showed higher response after the application of anti-CD25 when compared to animals that did not receive the antibody (Fig. 5A). This effect was observed for both the PADRE.Sox2opt-cyt and Sox2opt-cyt plasmids. Furthermore, an enhanced PADRE-
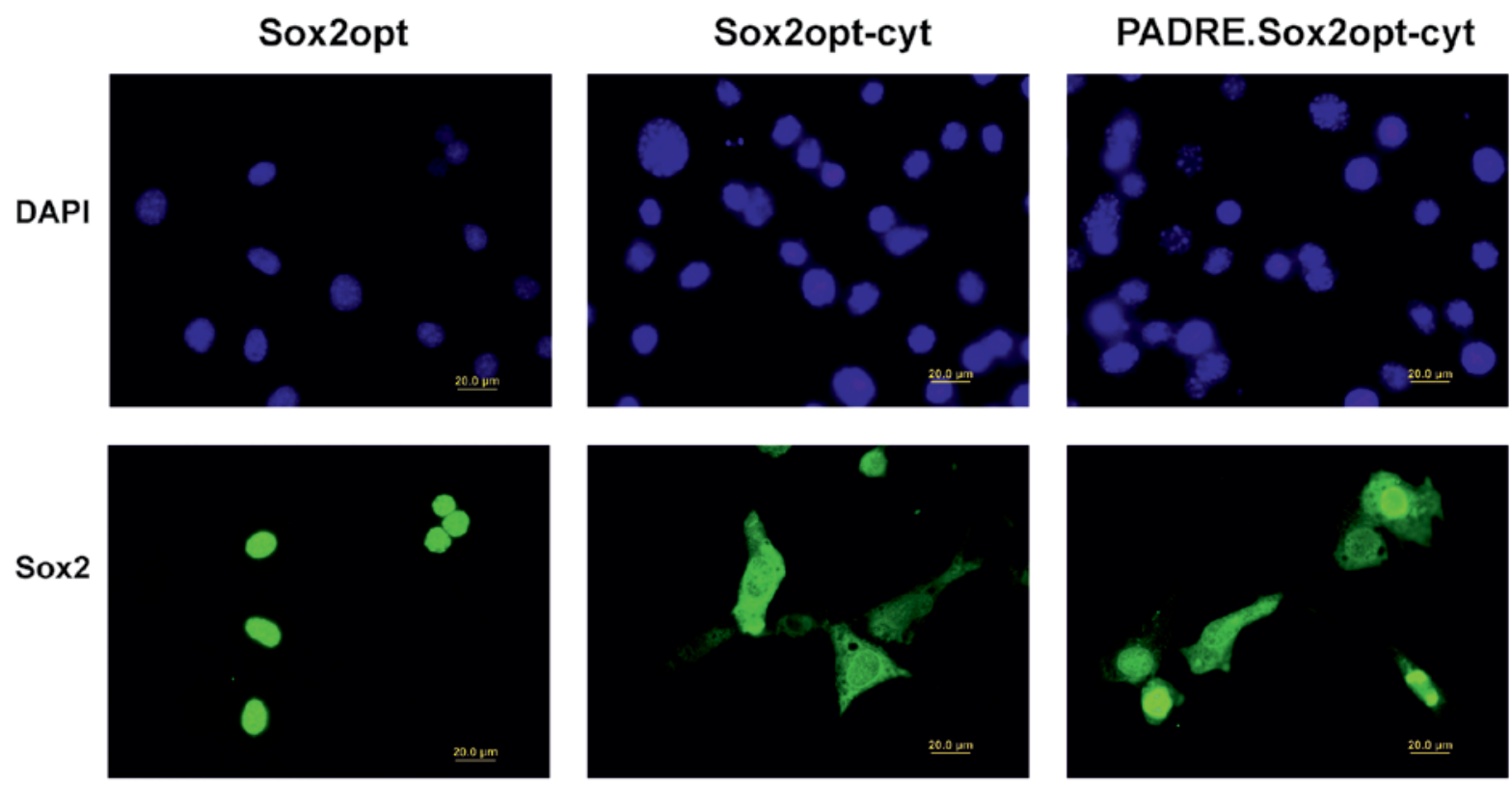

Figure 3. Cellular localization of the Sox 2 proteins. In transfected NIH/3T3 cells, Sox2 localization was detected by immunofluorescence staining. 
A

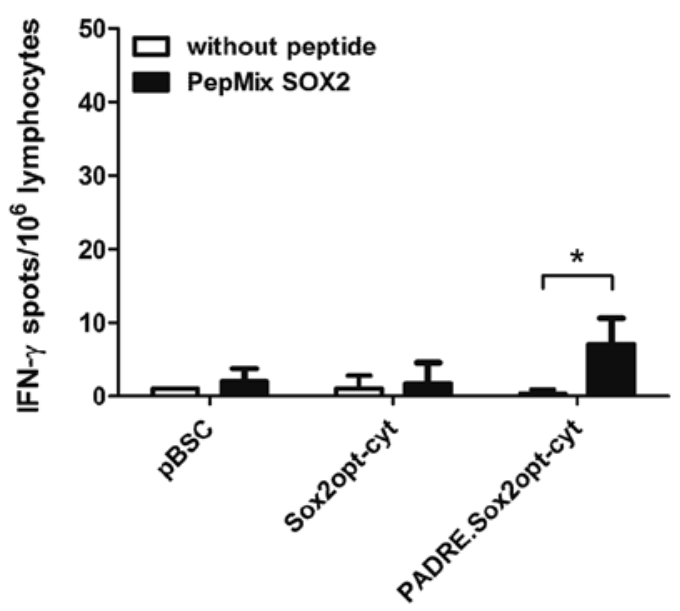

B

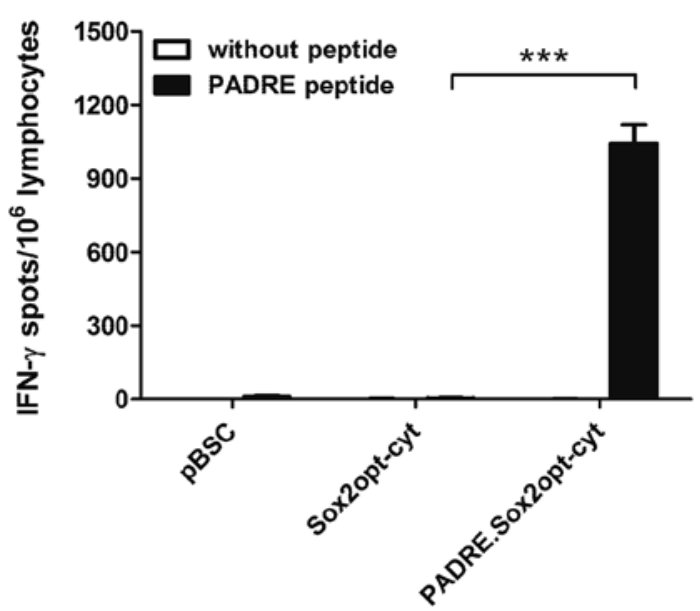

Figure 4. Immunogenicity of Sox 2 genes. Mice were immunized three times by DNA vaccines. Mononuclear cells were prepared from pooled splenocytes of immunized mice and incubated with PepMix SOX2 (A) or the PADRE peptide (B). Control cells were cultured without peptides. IFN- $\gamma$ producing cells were detected by an ELISPOT assay. Columns, mean of triplicate samples; bars, $\pm \mathrm{SD} ;{ }^{*} \mathrm{P}<0.05 ;{ }^{* * *} \mathrm{P}<0.0001$.

A

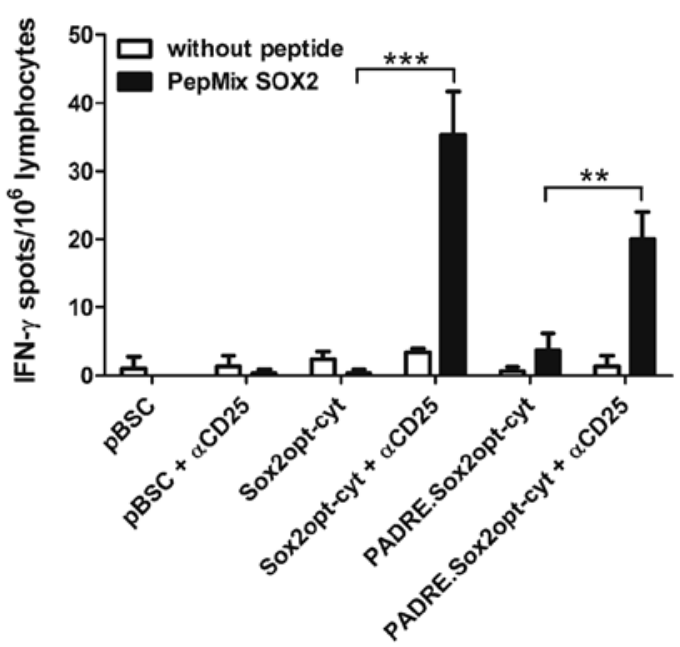

B

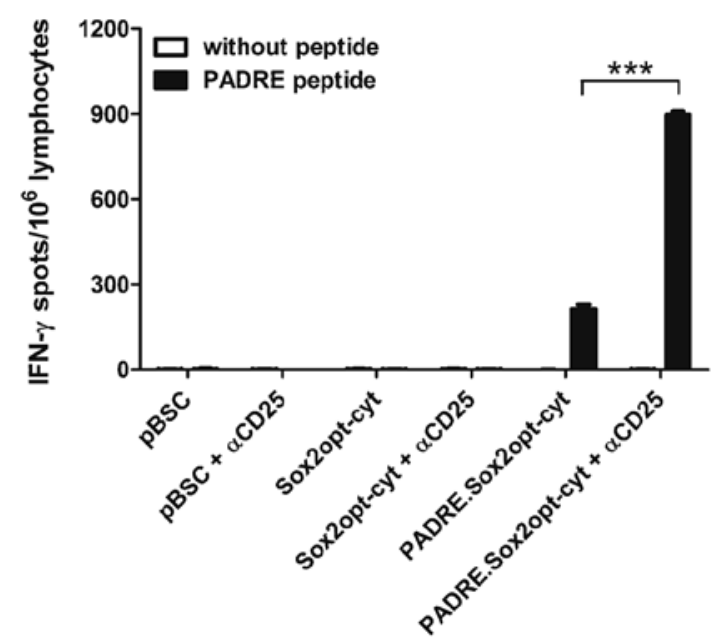

Figure 5. Efficacy of DNA vaccines after depletion of Treg cells. Mice were immunized three times by DNA vaccines. Anti-CD25 antibody was injected into some mice before the first immunization. Mononuclear cells were prepared from pooled splenocytes of immunized mice and incubated with PepMix SOX2 (A) or the PADRE peptide (B). Control cells were cultured without peptides. IFN- $\gamma$ producing cells were detected by an ELISPOT assay. Columns, mean of triplicate samples; bars, $\pm \mathrm{SD} ;{ }^{* *} \mathrm{P}<0.01 ;{ }^{* * *} \mathrm{P}<0.001$.

specific immune response was found after depletion of Treg cells in mice immunized with the PADRE.Sox2opt-cyt construct (Fig. 5B).

The antitumor effect of DNA vaccination. Our screening of mouse tumor cell lines (B16-F10, NIH/3T3, TRAMP-C2, MK16/ABC, TC-1, A20, 12B1, and B210) by RT-PCR revealed that only TC-1 cells expressed the Sox 2 gene (data not shown). The production of the Sox 2 protein was confirmed by immunoblot (Fig. 6A) and immunofluorescence analyses (Fig. 6B). However, immunofluorescence staining found Sox 2 in nuclei of only $\sim 15 \%$ of TC-1 cells. Therefore, the production of the Sox 2 protein was also detected in seven clones derived from tumors induced by TC- 1 cells in mice immunized against the HPV16 E7 oncoprotein $(26,29)$. In five of them, including
TC-1/B7 clone (Fig. 6B), the Sox 2 production was substantially higher than in TC-1 cells and this protein was stained in all nuclei. In subsequent immunization experiments, TC-1/B7 cells were used to challenge mice, because these cells were highly sensitive to immunization against the E7 antigen (26).

The antitumor effect of immunization against Sox 2 was examined with the PADRE.Sox2opt-cyt plasmid. Simultaneously, the impact of Treg depletion with anti-CD25 delivered before immunization was tested. The PADRE.Sox2opt-cyt vaccine significantly inhibited the growth of TC-1/B7 tumors (Fig. 7). This effect was also recorded after the depletion of Treg cells (even at a higher level) but the combination of immunization and Treg depletion did not further enhance the antitumor response. 
A

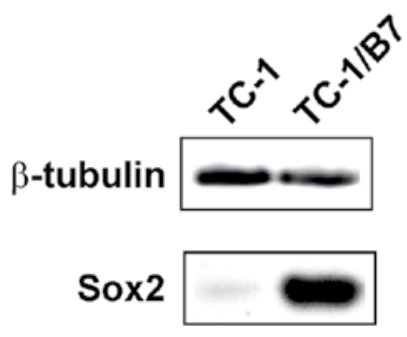

B

TC-1
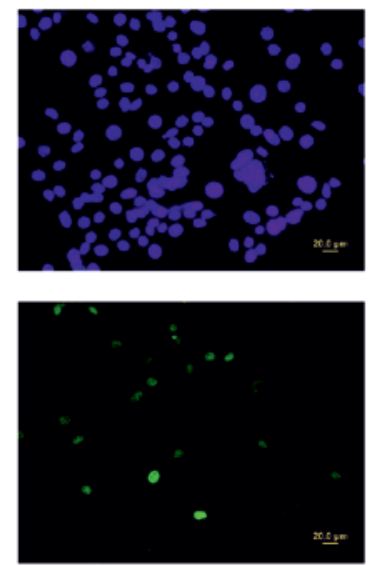

TC-1/B7
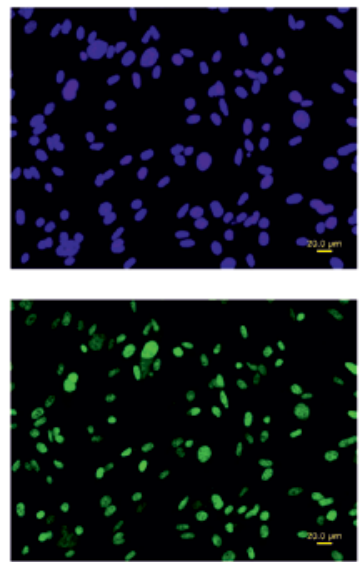

Figure 6. Sox 2 production in mouse TC-1 and TC-1/B7 tumor cell lines. (A) Cells analyzed by immunoblot staining. $\beta$-tubulin was stained to monitor protein load. (B) Cells analyzed by immunofluorescence staining. Cell nuclei were visualized using DAPI.

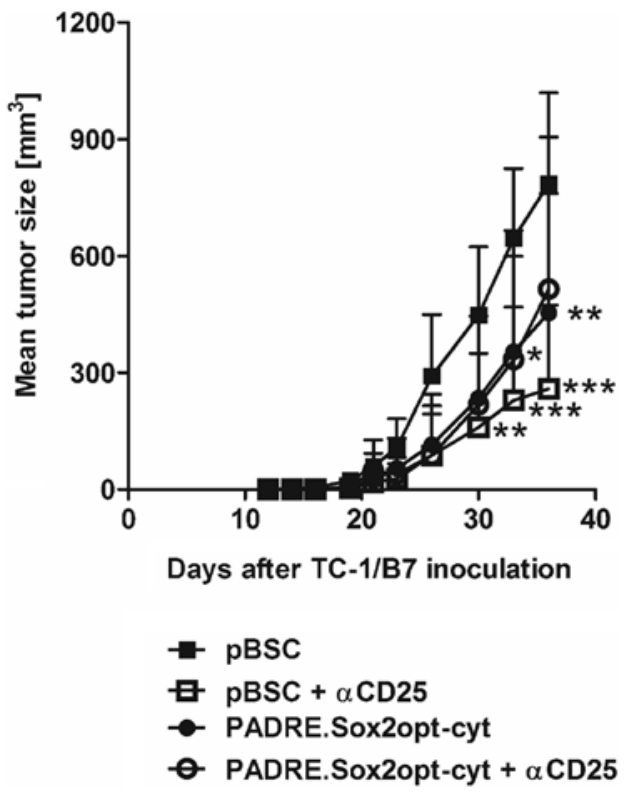

Figure 7. Antitumor effect of DNA vaccination. Mice were immunized three times with DNA vaccines. Some animals were injected with anti-CD25 before the first immunization. One week after the last immunization, TC-1/B7 cells were s.c. inoculated. Tumor growth was monitored twice a week. Bars, \pm SD; ${ }^{*} \mathrm{P}<0.05 ;{ }^{* *} \mathrm{P}<0.01 ;{ }^{* * *} \mathrm{P}<0.001$.

\section{Discussion}

The immunization against cancer cells could be a supportive treatment to synergize with other anticancer therapies. It can play a role especially in the elimination of CSCs, thus preventing recurrences and curing minimal residual disease. To target CSCs by vaccination, suitable antigens need to be identified. However, seeking for markers specific for CSCs suggested the difficulty of such efforts. The similarity of transcriptional profiles between ESCs and CSCs pointed to pluripotency transcription factors as candidate antigens.

In this study, we chose one of them, the Sox 2 protein, for the development of an experimental DNA vaccine. To enhance vaccine efficacy, we first ordered the synthesis of a codon-optimized gene which usually results in increased production of a protein. However, the steady-state level of the optimized Sox2 (Sox2opt) was similar to that of the wt Sox2. Such result is not exceptional as of 50 proteins analyzed for codon optimization in a study using the GeneOptimizer expert software, seven (14\%) did not exhibit augmented synthesis (30). Nevertheless, we used the Sox2opt gene for further construction of the DNA vaccine, because the modification of codons could prevent a potential homologous recombination into the host genome. Next, as Sox 2 has oncogenic potential (20), we mutated both NLSs in Sox 2 to abolish its transcriptional activity. In accordance with the previous study (19), these mutations did not completely inhibit nuclear localization. However, the mutagenesis of NLSs not only affected Sox 2 cellular localization, but also directly reduced its transcriptional activity and inhibited its cooperation with Oct4 and wt Sox2 (19). Both our alterations of Sox 2, the codon optimization at the level of the DNA sequence, and the NLS abolition at the level of the protein sequence, enhance the safety of the DNA vaccine. Finally, we added the sequence encoding the PADRE helper epitope to the 5' terminus of the Sox2opt-cyt gene. In our previous study (31), this epitope was more potent than the universal p30 helper epitope from the tetanus toxin and induced strong Th1 immunity that markedly enhanced activation of CD8 ${ }^{+}$ T lymphocytes.

To detect the immune response against mouse Sox 2 in an ELISPOT assay, we first performed computational prediction of $\mathrm{H}-2^{\mathrm{b}}$ and $\mathrm{H}-2^{\mathrm{d}}$ epitopes and tested the reactivity of five synthetic nonapeptides representing the predicted epitopes (aa 42-50, 75-83, 91-99, 111-119 and 125-133) with splenocytes obtained after immunization of C57BL/6 and BALB/c mice. However, none of these peptides was active in these examinations (data not shown). Therefore, we also tested the stimulation of lymphocytes with PepMix SOX2 containing 77 peptides derived from the human SOX 2 protein. As homology between mouse and human proteins is $\sim 97 \%$, most mouse epitopes should be preserved in PepMix SOX2. Indeed, we found significant Sox2-specific activation of lymphocytes 
with PepMix SOX2, but only after immunization of C57BL/6 mice with the PADRE.Sox2opt-cyt. This result suggests that the Th1 immunity induced by the PADRE epitope was necessary for breaking tolerance to the Sox 2 self-antigen.

The depletion of Treg cells enhanced the efficacy of DNA vaccination against various antigens (32-34) and resulted in broken tolerance (34). Therefore, we used antibody against CD25 for Treg depletion and showed the augmentation of both PADRE- and Sox2-specific responses. In our analogous study with a DNA vaccine against legumain overexpressed in tumor macrophages, we found enhanced immunity against legumain only after vaccination with the fusion gene carrying the sequence encoding the p30 helper epitope (Smahel et al, unpublished data). However, the response against Sox 2 was also increased after immunization with the Sox2opt-cyt gene without PADRE. These results propose that the induction of Th immunity was necessary for the effect of Treg depletion and that Sox 2 contains a strong helper epitope. The latter conclusion is supported by studies detecting SOX2-specific immunity in patients with premalignant or malignant diseases, because both $\mathrm{CD}^{+}$and $\mathrm{CD}^{+}{ }^{+}$reactive $\mathrm{T}$ cells were demonstrated $(8,35)$.

Of eight mouse oncogenic cell lines of various origins, Sox 2 expression was only found in TC-1 cells derived from the lung. When clones isolated from a tumor induced with TC-1 cells in immunized mice were examined, five out of seven clones produced a markedly higher amount of the Sox 2 protein. Noh et al (36) have also demonstrated Sox 2 expression in TC-1 cells, but they found the same expression in immunoresistant TC-1 P3 cells derived from tumors after 3 serial passages in immunized mice.

For the examination of the antitumor effect of DNA vaccination against Sox 2, we used TC-1/B7 cells that were highly sensitive to immunization against the HPV16 E7 oncoprotein (26). However, despite strong production of Sox2, DNA vaccination against this self-antigen did not prevent tumor development. Otherwise, it significantly reduced tumor growth, but this impact was low. Tumor growth of TC-1/B7 cells was also significantly inhibited by the depletion of Treg cells, but the combination of immunization and depletion did not further strengthen the antitumor effect. We obtained a similar result after immunization against legumain. Treg lymphocytes usually support tumor growth by their immunosupressive activities, but in tumors with intense inflammation that is useful for tumor progression, they can reduce this inflammation and thus inhibit tumor development (37). It could also be the case of tumors in immunized mice with a high Th1 response.

In conclusion, we constructed an experimental DNA vaccine against Sox 2 and demonstrated in vitro the enhancement of the Sox2-specific response by the addition of the PADRE helper epitope and depletion of Treg cells. However, this depletion did not augment the antitumor effect. Our results support the notion (37) that the elimination of Treg cells, e.g. by antibodies against PD-1 or PD-L1 in clinical trials, may not always be beneficial in combination with other types of immunotherapy.

\section{Acknowledgements}

We thank P. Vesela and K. Kernova for technical assistance. This study was supported by grant NT11541-4/2010 from the Ministry of Health of the Czech Republic and grants CZ.2.16/3.1.00/24001 and CZ.2.16/3.1.00/28007 from the European Regional Development Fund, Operational Programme Prague-Competitiveness.

\section{References}

1. Sugihara E and Saya H: Complexity of cancer stem cells. Int J Cancer 132: 1249-1259, 2013.

2. Xu Q, Liu G, Yuan X, Xu M, Wang H, Ji J, Konda B, Black KL and Yu JS: Antigen-specific T-cell response from dendritic cell vaccination using cancer stem-like cell-associated antigens. Stem Cells 27: 1734-1740, 2009.

3. Wong DJ, Liu H, Ridky TW, Cassarino D, Segal E and Chang HY: Module map of stem cell genes guides creation of epithelial cancer stem cells. Cell Stem Cell 2: 333-344, 2008.

4. Muller FJ, Laurent LC, Kostka D, Ulitsky I, Williams R, Lu C, Park IH, Rao MS, Shamir R, Schwartz PH, Schmidt NO and Loring JF: Regulatory networks define phenotypic classes of human stem cell lines. Nature 455: 401-405, 2008.

5. Ben-Porath I, Thomson MW, Carey VJ, Ge R, Bell GW, Regev A and Weinberg RA: An embryonic stem cell-like gene expression signature in poorly differentiated aggressive human tumors. Nat Genet 40: 499-507, 2008.

6. Boyer LA, Lee TI, Cole MF, Johnstone SE, Levine SS, Zucker JP, Guenther MG, Kumar RM, Murray HL, Jenner RG, Gifford DK, Melton DA, Jaenisch R and Young RA: Core transcriptional regulatory circuitry in human embryonic stem cells. Cell 122: 947-956, 2005.

7. Gure AO, Stockert E, Scanlan MJ, Keresztes RS, Jager D, Altorki NK, Old LJ and Chen YT: Serological identification of embryonic neural proteins as highly immunogenic tumor antigens in small cell lung cancer. Proc Natl Acad Sci USA 97: 4198-4203, 2000.

8. Spisek R, Kukreja A, Chen LC, Matthews P, Mazumder A, Vesole D, Jagannath S, Zebroski HA, Simpson AJ, Ritter G, Durie B, Crowley J, Shaughnessy JD Jr, Scanlan MJ, Gure AO, Barlogie B and Dhodapkar MV: Frequent and specific immunity to the embryonal stem cell-associated antigen SOX2 in patients with monoclonal gammopathy. J Exp Med 204: 831-840, 2007

9. Schmitz M, Temme A, Senner V, Ebner R, Schwind S, Stevanovic S, Wehner R, Schackert G, Schackert HK, Fussel M, Bachmann M, Rieber EP and Weigle B: Identification of SOX2 as a novel glioma-associated antigen and potential target for T cell-based immunotherapy. Br J Cancer 96: 1293-1301, 2007.

10. Laga AC, Lai CY, Zhan Q, Huang SJ, Velazquez EF, Yang Q, Hsu MY and Murphy GF: Expression of the embryonic stem cell transcription factor SOX2 in human skin: relevance to melanocyte and merkel cell biology. Am J Pathol 176: 903-913, 2010.

11. Zhang X, Yu H, Yang Y, Zhu R, Bai J, Peng Z, He Y, Chen L, Chen W, Fang D, Bian X and Wang R: SOX2 in gastric carcinoma, but not Hath1, is related to patients' clinicopathological features and prognosis. J Gastrointest Surg 14: 1220-1226, 2010.

12. Lengerke C, Fehm T, Kurth R, Neubauer H, Scheble V, Muller F, Schneider F, Petersen K, Wallwiener D, Kanz L, Fend F, Perner S, Bareiss PM and Staebler A: Expression of the embryonic stem cell marker SOX2 in early-stage breast carcinoma. BMC Cancer 11: 42, 2011.

13. Bass AJ, Watanabe H, Mermel CH, Yu S, Perner S, Verhaak RG, Kim SY, Wardwell L, Tamayo P, Gat-Viks I, Ramos AH, Woo MS, Weir BA, Getz G, Beroukhim R, O'Kelly M, Dutt A, Rozenblatt-Rosen O, Dziunycz P, Komisarof J, Chirieac LR, Lafargue CJ, Scheble V, Wilbertz T, Ma C, Rao S, Nakagawa H, Stairs DB, Lin L, Giordano TJ, Wagner P, Minna JD, Gazdar AF, Zhu CQ, Brose MS, Cecconello I, Jr UR, Marie SK, Dahl O, Shivdasani RA, Tsao MS, Rubin MA, Wong KK, Regev A, Hahn WC, Beer DG, Rustgi AK and Meyerson M: SOX2 is an amplified lineage-survival oncogene in lung and esophageal squamous cell carcinomas. Nat Genet 41: 1238-1242, 2009.

14. Yuan P, Kadara H, Behrens C, Tang X, Woods D, Solis LM, Huang J, Spinola M, Dong W, Yin G, Fujimoto J, Kim E, Xie Y, Girard L, Moran C, Hong WK, Minna JD and Wistuba II: Sex determining region Y-Box 2 (SOX2) is a potential cell-lineage gene highly expressed in the pathogenesis of squamous cell carcinomas of the lung. PLoS One 5: e9112, 2010. 
15. Hussenet T, Dali S, Exinger J, Monga B, Jost B, Dembele D, Martinet N, Thibault C, Huelsken J, Brambilla E and du Manoir S: SOX2 is an oncogene activated by recurrent $3 \mathrm{q} 26.3$ amplifications in human lung squamous cell carcinomas. PLoS One 5: e8960, 2010.

16. Freier K, Knoepfle K, Flechtenmacher C, Pungs S, Devens F, Toedt G, Hofele C, Joos S, Lichter P and Radlwimmer B: Recurrent copy number gain of transcription factor SOX2 and corresponding high protein expression in oral squamous cell carcinoma. Genes Chromosomes Cancer 49: 9-16, 2010.

17. Maier S, Wilbertz T, Braun M, Scheble V, Reischl M, Mikut R, Menon R, Nikolov P, Petersen K, Beschorner C, Moch H, Kakies C, Protzel C, Bauer J, Soltermann A, Fend F, Staebler A, Lengerke $\mathrm{C}$ and Perner S: SOX2 amplification is a common event in squamous cell carcinomas of different organ sites. Hum Pathol 42: 1078-1088, 2011.

18. Wilbertz T, Wagner P, Petersen K, Stiedl AC, Scheble VJ, Maier S, Reischl M, Mikut R, Altorki NK, Moch H, Fend F, Staebler A, Bass AJ, Meyerson M, Rubin MA, Soltermann A, Lengerke C and Perner S: SOX2 gene amplification and protein overexpression are associated with better outcome in squamous cell lung cancer. Mod Pathol 24: 944-953, 2011.

19. Li J, Pan G, Cui K, Liu Y, Xu S and Pei D: A dominant-negative form of mouse SOX2 induces trophectoderm differentiation and progressive polyploidy in mouse embryonic stem cells. J Biol Chem 282: 19481-19492, 2007.

20. Liu K, Lin B, Zhao M, Yang X, Chen M, Gao A, Liu F, Que J and Lan X: The multiple roles for Sox 2 in stem cell maintenance and tumorigenesis. Cell Signal 25: 1264-1271, 2013.

21. Dhodapkar MV and Dhodapkar KM: Spontaneous and therapyinduced immunity to pluripotency genes in humans: clinical implications, opportunities and challenges. Cancer Immunol Immunother 60: 413-418, 2011.

22. Smahel M, Sima P, Ludvikova V and Vonka V: Modified HPV16 E7 genes as DNA vaccine against E7-containing oncogenic cells. Virology 281: 231-238, 2001.

23. Alexander J, Sidney J, Southwood S, Ruppert J, Oseroff C, Maewal A, Snoke K, Serra HM, Kubo RT and Sette A: Development of high potency universal DR-restricted helper epitopes by modification of high affinity DR-blocking peptides. Immunity 1: 751-761, 1994.

24. Jainchill JL, Aaronson SA and Todaro GJ: Murine sarcoma and leukemia viruses: assay using clonal lines of contact-inhibited mouse cells. J Virol 4: 549-553, 1969.

25. Lin KY, Guarnieri FG, Staveley OK, Levitsky HI, August JT, Pardoll DM and Wu TC: Treatment of established tumors with a novel vaccine that enhances major histocompatibility class II presentation of tumor antigen. Cancer Res 56: 21-26, 1996.
26. Smahel M, Smahelova J, Tejklova P, Tachezy R and Marinov I: Characterization of cell lines derived from tumors induced by TC-1 cells in mice preimmunized against HPV16 E7 oncoprotein. Int J Oncol 27: 731-742, 2005.

27. Kaufmann AM, Gissmann L, Schreckenberger C and Qiao L: Cervical carcinoma cells transfected with the CD80 gene elicit a primary cytotoxic T lymphocyte response specific for HPV 16 E7 antigens. Cancer Gene Ther 4: 377-382, 1997.

28. Sudbeck P and Scherer G: Two independent nuclear localization signals are present in the DNA-binding high-mobility group domains of SRY and SOX9. J Biol Chem 272: 27848-27852, 1997.

29. Smahel M, Sima P, Ludvikova V, Marinov I, Pokorna D and Vonka V: Immunisation with modified HPV16 E7 genes against mouse oncogenic TC-1 cell sublines with downregulated expression of MHC class I molecules. Vaccine 21: 1125-1136, 2003.

30. Fath S, Bauer AP, Liss M, Spriestersbach A, Maertens B, Hahn P, Ludwig C, Schafer F, Graf M and Wagner R: Multiparameter RNA and codon optimization: a standardized tool to assess and enhance autologous mammalian gene expression. PLoS One 6: e17596, 2011.

31. Smahel M,Polakova I,Duskova M,Ludvikova V and Kastankova I: The effect of helper epitopes and cellular localization of an antigen on the outcome of gene gun DNA immunization. Gene Ther 21: 225-232, 2014.

32. Furuichi Y, Tokuyama H, Ueha S, Kurachi M, Moriyasu F and Kakimi K: Depletion of CD25 ${ }^{+} \mathrm{CD} 4^{+} \mathrm{T}$ cells (Tregs) enhances the $\mathrm{HBV}$-specific $\mathrm{CD} 8^{+} \mathrm{T}$ cell response primed by DNA immunization. World J Gastroenterol 11: 3772-3777, 2005.

33. Chuang CM, Hoory T, Monie A, Wu A, Wang MC and Hung CF: Enhancing therapeutic HPV DNA vaccine potency through depletion of $\mathrm{CD} 4^{+} \mathrm{CD} 25^{+} \mathrm{T}$ regulatory cells. Vaccine 27: 684-689, 2009.

34. Jacob JB, Kong YC, Nalbantoglu I, Snower DP and Wei WZ: Tumor regression following DNA vaccination and regulatory $T$ cell depletion in neu transgenic mice leads to an increased risk for autoimmunity. J Immunol 182: 5873-5881, 2009.

35. Dhodapkar KM, Gettinger SN, Das R, Zebroski H and Dhodapkar MV: SOX2-specific adaptive immunity and response to immunotherapy in non-small cell lung cancer. Oncoimmunology 2: e25205, 2013.

36. Noh KH, Lee YH, Jeon JH, Kang TH, Mao CP, Wu TC and Kim TW: Cancer vaccination drives Nanog-dependent evolution of tumor cells toward an immune-resistant and stem-like phenotype. Cancer Res 72: 1717-1727, 2012.

37. Whiteside TL: Regulatory T cell subsets in human cancer: are they regulating for or against tumor progression? Cancer Immunol Immunother 63: 67-72, 2014. 